\title{
Decreased expression of FBXW7 by ERK1/2 activation in drug-resistant cancer cells confers transcriptional activation of MDR1 by suppression of ubiquitin degradation of HSF1
}

\author{
Gil-Im Mun ${ }^{1}$, Eun Choi ${ }^{1}$, Yeongmin Lee ${ }^{1}$ and Yun-Sil Lee $\mathbb{1}^{1}$
}

\begin{abstract}
The acquisition of MDR1-mediated chemoresistance poses a major obstacle to the success of conventional chemotherapeutic agents. HSF1 is also involved in chemoresistance, and several studies have demonstrated the relationship between HSF1 and MDR1 but without any consistent results. Paclitaxel- and doxorubicin-resistant cancer cells showed higher expression of MDR1 and HSF1. Depletion of HSF1 decreased mdr1 expression at mRNA level, and HSF1 directly interacted with the promoter site of $m d r 1$, suggesting its role as a transcriptional regulator of MDR1. Phosphorylation of Ser303/307, which was involved in protein stability of HSF1 by FBXW7-mediated degradation, was found to be important for transcriptional activation of $m d r 1$. Drug-resistant cells showed decreased expression of FBXW7, which was mediated by the activation of ERK1/2, thus indicating that over-activation of ERK1/2 in drugresistant cells decreased FBXW7 protein stability, which finally inhibited protein degradation of pHSF1 at Ser303/307. There was a positive correlation between immunofluorescence data of pHSF1 at Ser303/307 and MDR1 in carcinogeninduced rat mammary tumors and human lung cancers. These findings identified the post-translational mechanisms of HSF1 transcription in MDR1 regulation of drug resistance development.
\end{abstract}

\section{Introduction}

Heat-shock factor 1 (HSF1) is a master regulator of the heat-shock response and facilitates cell survival and proliferation in eukaryotes. It has been widely reported that HSF1 is often overexpressed in cancer cells, thus suggesting that it has a role in tumorigenesis. HSF1 is found to mediate the protection of cancer cells from programed cell death by overriding cell cycle checkpoints and thus exacerbating metastasis. Activation of HSF1-dependent stress response, a cytoprotective mechanism, may greatly influence the development of an adaptive and protective phenotype in cancer cells subjected to anticancer agents.

\footnotetext{
Correspondence: Yun-Sil Lee (yslee0425@ewha.ac.kr)

${ }^{1}$ Graduate School of Pharmaceutical Sciences, Ewha Womans University, Seoul 120-750, Korea

These authors contributed equally: Gil-Im Mun, Eun Choi

Edited by M.L. Asselin-labat
}

Elevated expression of heat-shock proteins (HSPs) has been reported in many types of human malignancies and is reportedly associated with resistance of cancer cells to apoptosis induced by chemotherapeutic agents ${ }^{1-3}$. In addition, HSP-independent mechanisms are also reportedly involved in HSF1-regulated resistance of cancer cells to chemotherapeutics ${ }^{4,5}$.

Post-translational modifications have fundamental roles in the activation and suppression of HSF1, including subcellular localization of HSF1 and interaction of HSF1 with partner proteins. Although the specific requirements for phosphorylation events and their specific roles in the regulation of HSF1 remain unclear, 12 phosphorylated serine residues have been identified ${ }^{6}$. Regarding phosphorylation, several serine residues appear to participate in the regulation of HSF1 transcription activity. Among these, phosphorylation of Ser230, Ser320, Ser326, and

\section{(c) The Author(s) 2020}

(c) (i) Open Access This article is licensed under a Creative Commons Attribution 4.0 International License, which permits use, sharing, adaptation, distribution and reproduction in any medium or format, as long as you give appropriate credit to the original author(s) and the source, provide a link to the Creative Commons license, and indicate if changes were made. The images or other third party material in this article are included in the article's Creative Commons license, unless indicated otherwise in a credit line to the material. If material is not included in the article's Creative Commons license and your intended use is not permitted by statutory regulation or exceeds the permitted use, you will need to obtain permission directly from the copyright holder. To view a copy of this license, visit http://creativecommons.org/licenses/by/4.0/. 
Ser419 contributes to activation of HSF1, whereas phosphorylation of Ser121, Ser303, Ser307, and Ser363 represses its activity ${ }^{7}$.

Previous studies have suggested that HSF1 activation is associated with poor outcome in breast cancer $^{8}$, and reduced FBXW7 expression or inactivating mutations is significantly correlated with poor patient prognosis in multiple cancers ${ }^{9}$. HSF1 accumulation due to altered expression of a substrate-targeting subunit of the SCF (Skp-1-Cull-F-box) ubiquitin ligase complex, FBXW7, provides an advantage in cancer cells during disease progression. FBXW7 targets several key regulators of proliferation, growth, and apoptosis for proteasomal degradation $^{10}$, and FBXW7 is mutated in a significant portion of diverse human cancers ${ }^{11-13}$. The interaction between FBXW7 and HSF1 is reported and FBXW7 controls the stability of nuclear HSF $1^{14}$.

The acquisition of the multidrug-resistance (MDR) phonotype, defined as increased resistance against cytotoxic drugs with unrelated structures, represents one of the major obstacles for chemotherapy of tumors and other malignancies. The MDR gene was involved in MDR phenotype ${ }^{15}$. Drug resistance is acquired by prolonged exposure to cytotoxic drugs with the amplification of the $m d r 1$ gene. The transcriptional regulation of $m d r 1$ is tissue specifically induced; however, the molecular mechanisms are not yet fully clarified. HSF1 can participate in $m d r 1$ gene expression. Heat-shock elements (HSE) have been identified in the $m d r 1$ gene promoter ${ }^{16,17}$, and typical stress inducers, such as heat shock and arsenite, which induce HSP gene expression, also induce $m d r 1$ gene expression in some cell types ${ }^{16,17}$. Some MDR cell lines exhibit constitutively high HSF1-DNA binding activity $^{18}$, and quercetin can inhibit the HSF1-HSE binding and $m d r 1$ gene expression in MDR cells ${ }^{19}$. However, some reports suggest that the activation of MDR expression by heat shock and other stressors may be mediated by DNA sequences and transcription factors besides HSE of HSF1 (refs. ${ }^{20-22}$ ).

Several reports have demonstrated the relationship between HSF1 and MDR1. However, the precise role of HSF1 on the expression of MDR1 remains unclear. Several studies have presented the evidence that HSF1 is often overexpressed in chemoresistant cancer cells and that it upregulates the transcription of $m d r 1$, thereby enhancing the efflux of drugs ${ }^{4}$. Conversely, other reports have suggested an interplay between HSF1 and nuclear factor $\kappa B \quad\left(N_{\kappa} B\right)^{23}$. Studies have shown that HSF1 suppresses NFkB activity by interrupting its nuclear binding on DNA. Silencing HSF1 may lead to higher $\mathrm{NF} \kappa \mathrm{B}$ expression and activity and may enhance antiapoptotic activity in cells ${ }^{24}$.

Paclitaxel is used in platinum-based doublet regimens as the first-line standard therapy for advanced NSCLC ${ }^{25}$.
Despite its widespread use, its clinical effectiveness is limited by the development of paclitaxel-resistant cancer cells, which eventually leads to poor prognosis and relapse $^{26,27}$. Various mechanisms involved in acquired paclitaxel resistance have been reported. The best understood mechanism of paclitaxel resistance involves the overexpression of MDR1, which is encoded by the $m d r 1$ gene conferring the multidrug-resistance phenotype $^{28}$. However, further understanding of precise mechanisms involved in paclitaxel resistance is greatly warranted.

In this study, chemotherapeutic agent (paclitaxel or doxorubicin)-resistant cancer cells showed high expression of MDR1 and increased protein stability of HSF1, which were related to the paclitaxel-mediated resistance. Moreover, the phosphorylation of HSF1 at Ser303/307, which controlled HSF1 protein stability by FBXW7mediated ubiquitin degradation, was involved in transcriptional activation of $m d r l$, which may affect drug resistance.

\section{Results}

Increased expression of HSF1 after paclitaxel treatment in drug-resistant cancer cells accompanied with transcriptional activation of the $m d r 1$ gene

To elucidate the involvement of HSF1 in drug resistance, paclitaxel-resistant A549 lung cancer cells (A549taxolR) were generated by sustained treatment with 100-nM paclitaxel to maintain the paclitaxel resistance phenotype $^{29}$. In the case of doxorubicin (T47D-doxR or MCF7-doxR)-resistant T47D and MCF7 cells, they were previously reported to be resistant to doxorubicin ${ }^{30,31}$. All the resistance cells of A549-taxolR, T47D-doxR, and MCF7-doxR showed resistance to paclitaxel treatment on caspase-3 or PARP1 cleavage detection and cell viability assays. $\mathrm{IC}_{50}$ values after paclitaxel treatment were $4.4 \pm$ $0.15 \mu \mathrm{M}$ for A549, $0.77 \pm 0.08 \mu \mathrm{M}$ for T47D, and $0.73 \pm$ $0.03 \mu \mathrm{M}$ for MCF7 cells (MTT assay after $24 \mathrm{~h}$ treatment of paclitaxel). The degree of resistance in drug-resistant cells after paclitaxel treatment was $23.3 \%$ for A549-taxolR, $29.9 \%$ for T47D-doxR, and 32\% for MCF7-doxR. A549taxolR was less sensitive to paclitaxel than T47D-doxR or MCF7-doxR (Supplementary Fig. 1). These resistant cells showed increased expression of HSF1 and MDR1, which confers the MDR phenotype. Moreover, increasing dose of paclitaxel treatment did not affect HSF1 expression in drug-resistant cells, whereas HSF1 expression after paclitaxel treatment was dose-dependently inhibited in control cells. MDR1 expression was the highest in MCF7doxR cells and the lowest in A549-taxolR cells (Fig. 1a). Reverse transcriptase PCR (RT-PCR) data revealed that the $m d r 1$ gene was overexpressed in both A549-taxolR and T47D-doxR cells; the hsf1 gene levels were not changed (Fig. 1b). Paclitaxel treatment affected mRNA of 




$m d r 1$ more in drug-resistant cells (Fig. 1c). Promoter activity of $m d r 1$ was increased in both A549-taxolR and T47D-doxR cells when compared with their parent cells (Fig. 1d), suggesting that chemotherapeutic drug-resistant cells showed increased expression of MDR1 and HSF1; MDR1 expression was regulated at a transcriptional level and HSF1 expression at a post-translational level.

To identify the possible relationship between HSF1 and MDR1, HSF1 was knockdowned to A549-taxolR and T47D-doxR cells using siRNA directed against HSF1 (siHSF1); this led to down-regulation of MDR1 at both protein and mRNA levels (Fig. 2a, b). Knockdown of $h s f 1$ by the CRISPR/Cas9 system or stable transfection of shRNA to A549-taxolR cells also showed decreased levels of both MDR1 protein and mRNA as well as $m d r 1$ promoter activity (Fig. 2c, Supplementary Fig. 2a). Paclitaxel treatment to siHSF1-transfected A549-taxolR cells showed decreased expression of MDR1 and increased apoptosis when compared with control siRNA-treated cells (Fig. 2d). Chromatin immunoprecipitation (Chip) assay revealed that HSF1 was more enriched in the promoter region of $m d r 1$ in drugresistant A549-taxolR cells than in control A549 cells. Moreover, depletion of $h s f 1$ by the CRISPR/Cas9 system 


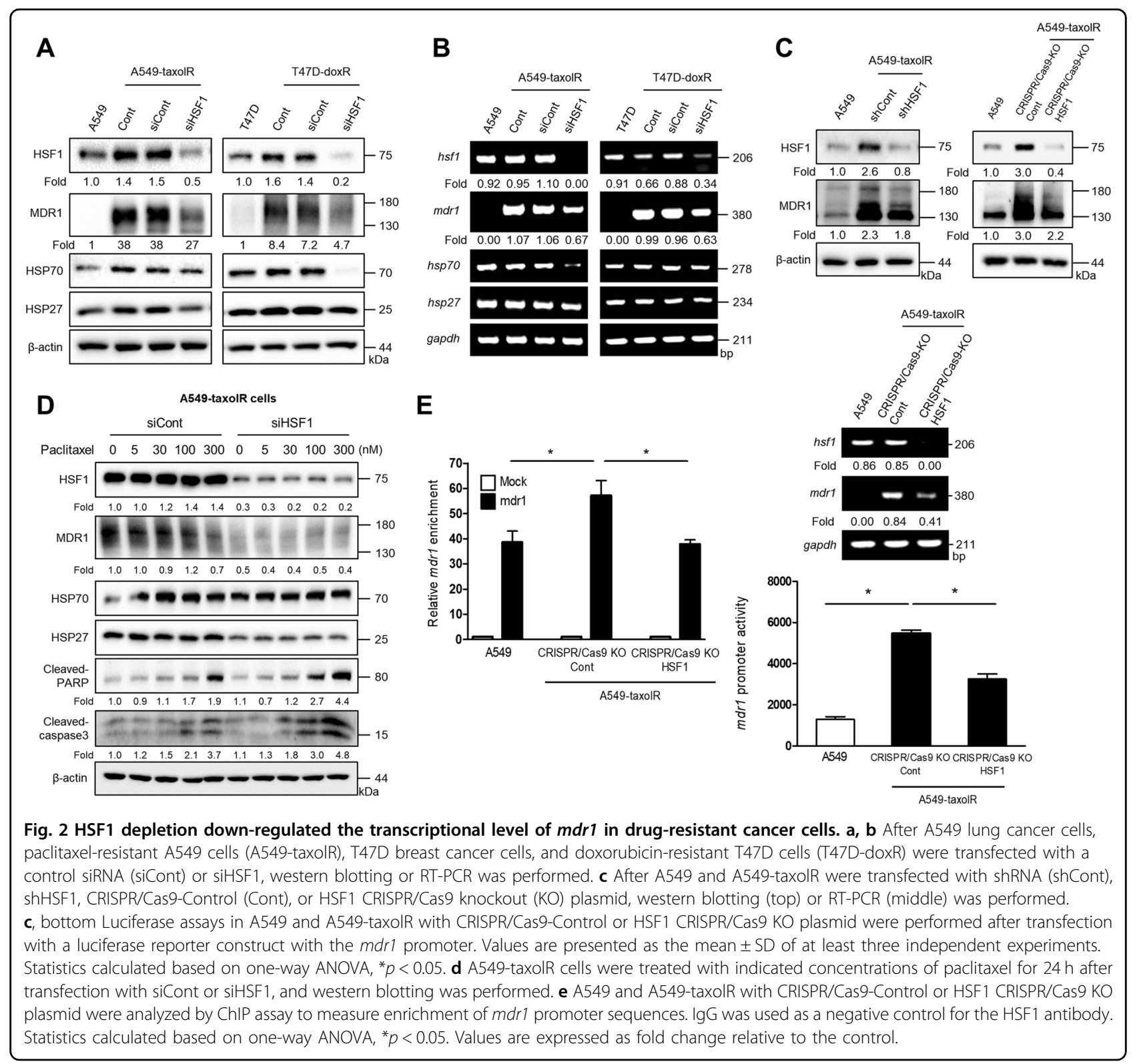

or stable transfection of shRNA to A549-taxolR significantly inhibited the enrichment of HSF1 in $m d r 1$ promoter region (Fig. 2e, Supplementary Fig. 2b). However, the CRISPR/Cas9 system to block the hsf1 expression did not completely inhibit MDR function, suggesting partial involvement of HSF1 in MDR regulation. In the case of knockdown of $m d r 1$ (siMDR1) to A549-taxolR and T47D-doxR cells, HSF1 expression at both mRNA and protein levels was not altered (Supplementary Fig. 3a, b). Moreover, wild-type (WT) $m d r 1$ overexpression to control A549 and T47D cells did not affect $h s f 1$ expression (Supplementary Fig. 3c), suggesting HSF1 as an upstream molecule for MDR1 overexpression in drug-resistant cells.

\section{Phosphorylation of HSF1 at Ser303/307 involved in transcriptional activation of $\mathbf{m d r} 1$}

Because hsf1 mRNA level was not changed in drugresistant cancer cells, post-translational modification of HSF1, such as phosphorylation patterns after paclitaxel treatment, was evaluated. Phosphorylation of Ser230 was slightly elevated and that of Ser326 was dramatically decreased in A549-taxolR cells when compared with control A549 cells. However, the phosphorylation of HSF1 at Ser303/307 was found to be dramatically increased in A549-taxolR cells, T47D-doxR cells, and MCF7-doxR cells. In control A549 and T47D cells, paclitaxel dose-dependent increase of HSF1 phosphorylation at Ser326 was found at $24 \mathrm{~h}$ of treatment; however, 


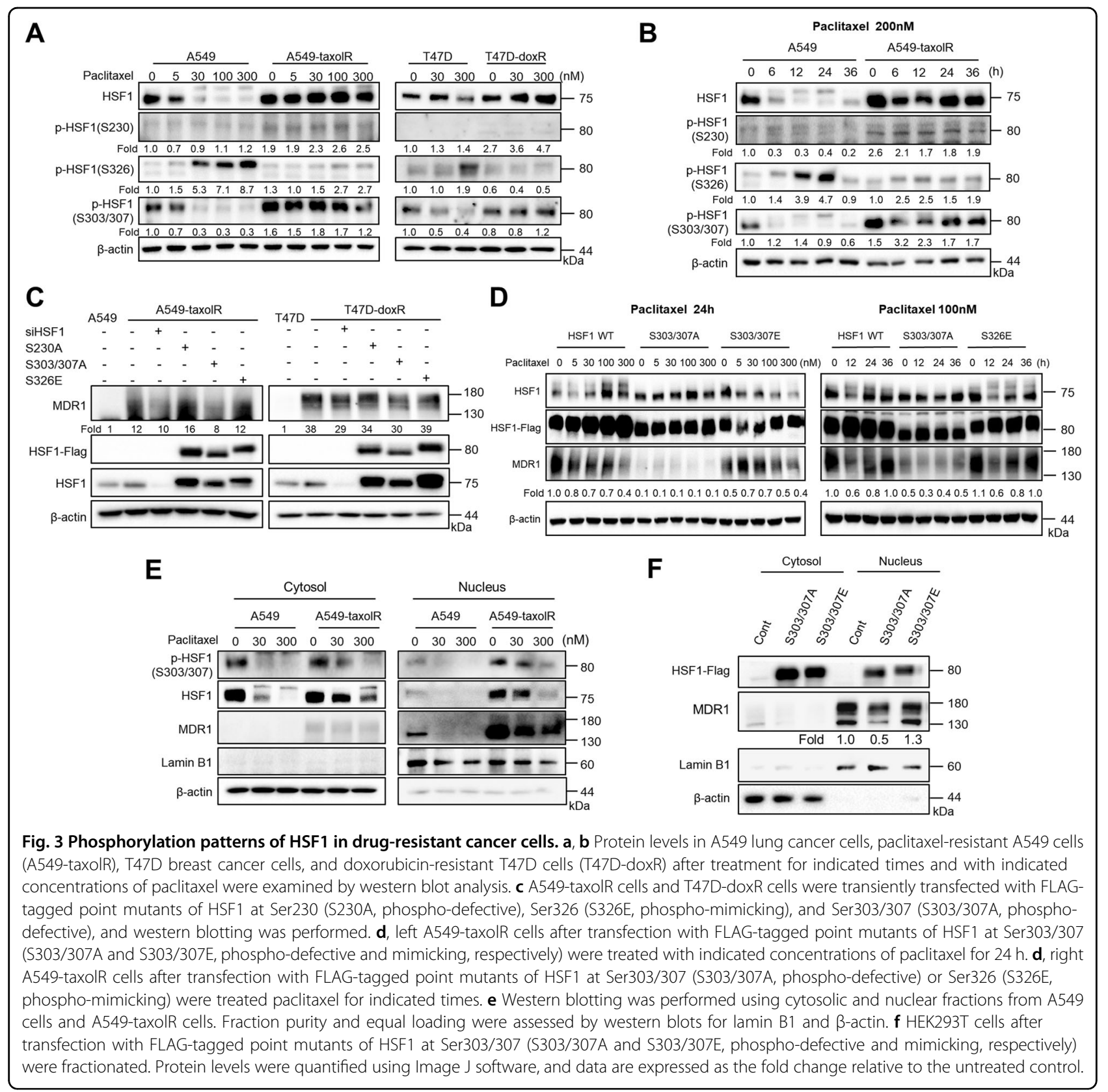

in drug-resistant cells, this increase was not found. HSF1 phosphorylation at Ser303/307 was inhibited by paclitaxel treatment in control cells; however, in drug-resistant cells, this inhibition was not found; paclitaxel did not affect the phosphorylation of HSF1 at Ser303/307 in drug-resistant cells (Fig. 3a, Supplementary Fig. 4a). Regarding other HSF1 phosphorylations, such as those at Ser216 or Ser419, these expressions were not evidently altered by $12 \mathrm{~h}$ paclitaxel treatment in drug-resistant cells (Supplementary Fig. 4b). HSF1 phosphorylation of Ser303/307 in A549-taxolR cells was slightly inhibited from 6 to $12 \mathrm{~h}$ of paclitaxel treatment; however, at $24 \mathrm{~h}$ of the treatment, it started recovering to a level comparable to that in paclitaxel untreated cells, whereas HSF1 phosphorylation of Ser303/307 in control cells was dramatically reduced from $6 \mathrm{~h}$ of paclitaxel treatment (Fig. 3b). Moreover, recovery after heat-shock treatment only affected the phosphorylation of HSF1 at Ser303/307 without any changes to phosphorylation sites of HSF1 at Ser230 and Ser326 in A549-taxolR cells (Supplementary Fig. 4c).

To examine direct correlation between the phosphorylation of HSF1 and MDR1 expression, A549-taxolR cells were transfected with HSF1 phosphorylation mutants. In the case of HSF1 at Ser303/307, both phospho-defective 
and phospho-active mutants (S303/307A and S303/307E, respectively) were used. Only the phospho-defective mutant S303/307A showed decreased MDR1 expression in both A549-taxolR and T47D-doxR cells. For mimicking unphosphorylated HSF1 at Ser230, S230A, the phosphodefective mutant form of HSF1, was transfected, and for mimicking phosphorylated HSF1 at Ser326, the phosphoactive mutant S326E was transfected, and these mutants did not affect MDR1 expression (Fig. 3c). We also treated A549-taxolR cells with paclitaxel and found that S303/ 307A showed decreased expression of MDR1 in terms of mRNA and protein levels, whereas S303/307E showed increased MDR1 expression. Another mutant of S326 did not affect MDR1 expression despite paclitaxel treatment (Fig. 3d, Supplementary Fig. 4d). However, when the expression of HSP70 was examined using mimicking and defective mutants of Ser303/307, a defective mutant of Ser303/307A showed increased expression of HSP70, whereas Ser303/307E showed vice versa (Supplementary Fig. 4e), which has already been reported ${ }^{32}$. HSF1 and pHSF1 at Ser303/307 were located not only in the cytosol but also in the nuclear fractions in drug-resistant A549taxolR cells, whereas in parent cells, they were mainly distributed in the cytosol. MDR1 localized mainly in the nucleus, and this was observed more in drug-resistant cells than in parent cells (Fig. 3e). When immunofluorescence staining for pHSF1 at Ser303/307 was performed, fluorescence intensity of pHSF1 at Ser303/307 in the nucleus was stronger in their drug-resistant A549taxolR cells than in their parent A549 cells (Supplementary Fig. 5a). To elucidate whether the phosphorylation of Ser303/307 affects the nuclear localization of HSF1, S303/ 307A and S303/307E were transfected to HEK293T cells, and nuclear localization was examined. Even though the distribution of S303/307A and S303/307E was similar in the cytosol and nucleus, only S303/307A inhibited MDR1 expression in the nucleus (Fig. 3f).

\section{Down-regulation of FBXW7 in drug-resistant cells involved in the inhibition of ubiquitin degradation of \\ phosphorylated HSF1 at Ser303/307}

Because sustained phosphorylation of HSF1 at Ser303/ 307 was involved in MDR expression in drug-resistant cells, we examined the protein stability of HSF1 according to the phosphorylation status of Ser303/307. Treatment with MG132, a proteasome inhibitor, increased the stability of pHSF1 at Ser303/307 in A549 cells; however, no significant difference was seen in A549-taxolR cells (Fig. 4a). To elucidate the mechanisms involved in increased protein stability of pHSF1 at Ser303/307 in drug-resistant cells, upstream signaling was investigated; it was found that FBXW7, a ubiquitin E3 ligase which reportedly interacts with pHSF1 at Ser303/307 and induces HSF1 degradation ${ }^{14}$, was down-regulated in
A549-taxolR cells and T47D-doxR cells even after paclitaxel treatment (Figs. 4b and 5a). However, the mRNA level of $f b x w 7$ was not altered (Supplementary Fig. 5b). Stably knockdowned $f b x w 7$ with shRNA FBXW7 to A549 cells increased pHSF1 at Ser303/307 and the stability of HSF1, which resulted in increased MDR1 protein expression. Overexpression of FBXW7 in A549-taxolR and HSF1 $+/+$ MEF cells led to enhanced proteosomal degradation of HSF1 by its direct binding to pHSF1 at Ser303/307, which resulted in reduced MDR1 expression (Fig. 4c, Supplementary Fig. 5c). Because pFBXW7 at T205 is involved in FBXW7 degradation ${ }^{33}$, we prepared a phospho-defective mutant of T205 (T205A). The transfection of T205A resulted in decreased stability of HSF1 and pHSF1 at Ser303/307 as well as decreased MDR1 expression. However, the expression of pHSF1 at Ser326 was not affected by T205A transfection to A549-taxolR cells. Moreover, T205A transfection to A549-taxolR cells showed increased expression of cleaved caspase- 3 and cleaved PARP1, even without paclitaxel treatment (Fig. 4d). Increased protein stability of S303/307E after CHX treatment was noted in A549-taxolR cells when compared with that in parent A549 cells (Fig. 4e). Transfection of shFBXW7, FBXW7-WT, or T205A did not alter HSP27 expression (Fig. 4c, d), suggesting that the protein degradation of pHSF 1 at Ser303/307 by FBXW7 is independent of its transcriptional role for HSPs.

\section{Activation of ERK $1 / 2$ in drug-resistant cells involved in decreased expression of FBXW7}

ERK1/2 activation was frequently observed in various drug-resistant cells ${ }^{34-36}$, and ERK1/2 activation induces FBXW7 phosphorylation at T205, which is involved in FBXW7 degradation ${ }^{33}$. FBXW7 deficiency or loss-of function confers resistance to chemotherapeutics, such as paclitaxel $^{37}$. Moreover, FBXW7 increased ubiquitin degradation of pHSF1 at Ser303/307 (ref. ${ }^{14}$ ). Indeed, A549-taxolR cells and T47D-doxR cells showed increased activation of ERK1/2 accompanied with down-regulation of the FBXW7 protein. When HSF1+/+ and HSF1-/MEF cells were compared, similar effects were observed (Fig. 5a). To elucidate the involvement of ERK1/2 activation in the expression of FBXW7, the resistant cells were treated with U0126 (an ERK1/2 inhibitor); consequently, we noted restored FBXW7 expression accompanied with degradation of pHSF1 at Ser303/307, thus resulting in characteristics very similar to those of control A549 cells. In the case of the expression of pHSF1 at Ser326, no alteration by ERK1/2 inhibitor was observed. When HSF1+/+ and HSF1-/- MEF cells were compared, similar phenomena were observed (Fig. 5b). Moreover, on treatment of drug-resistant cells with ERK1/2 inhibitor, increased ubiquitination of HSF1 and decreased protein stability of pHSF1 at Ser303/307 were detected (Fig. 5c), 


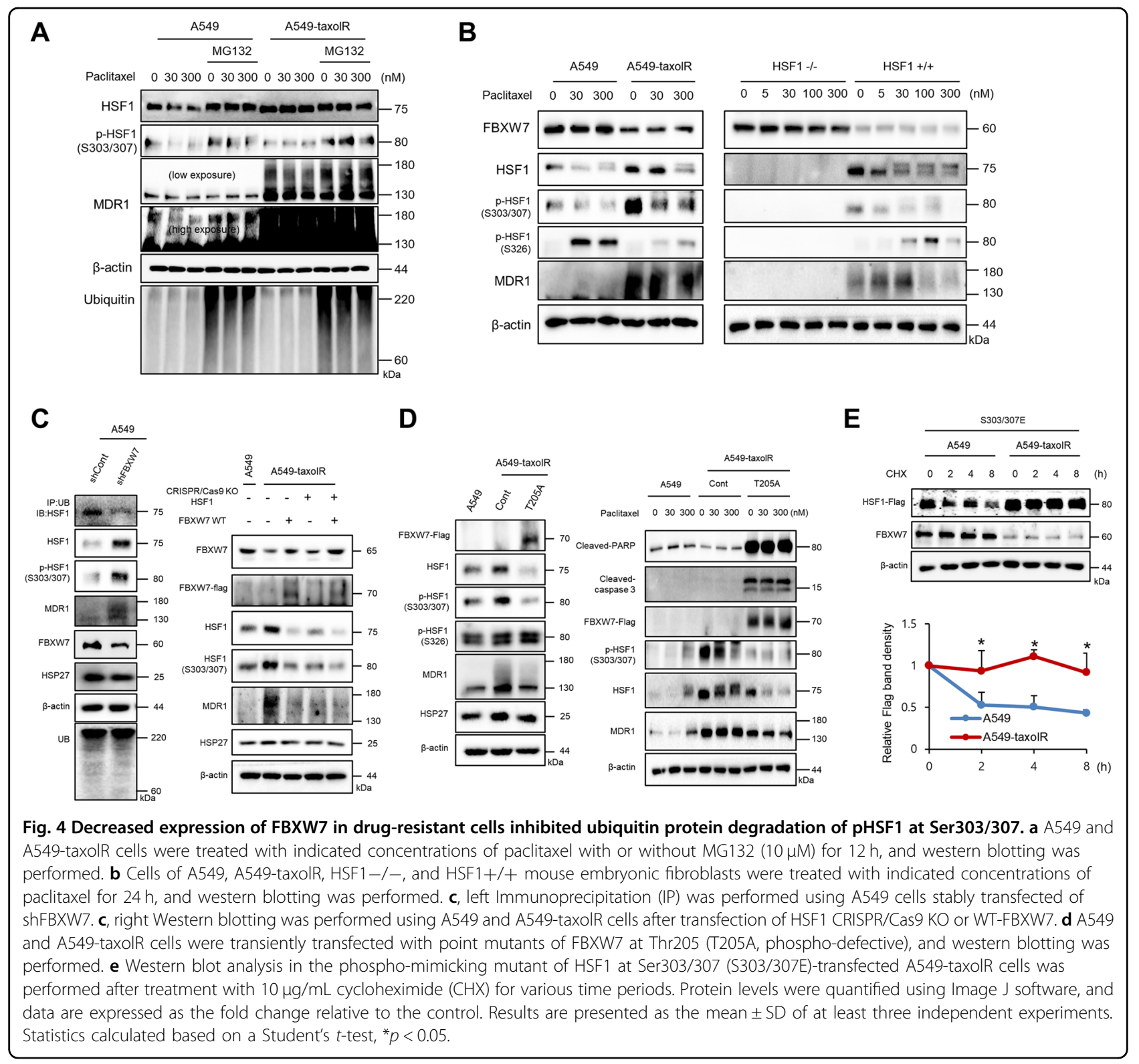

with sensitization to paclitaxel, when cleavage of PARP1 and cell survival were detected (Fig. 5d).

\section{Phosphorylation of HSF1 at Ser303/307 decreased paclitaxel-mediated cell death}

Because drug-resistant cells showed increased pHSF1 at Ser303/307 and increased MDR1 expression, the relationship between HSF1 phosphorylation of Ser303/307 and drug resistance was examined. The transfection of HSF1 WT or S303/307E to control A549 cells or T47D cells reduced PARP1 cleavage by paclitaxel treatment, and the levels were similar to those in A549-taxolR cells or T47D-doxR cells, respectively. However, the involvement of HSF1 in the development of paclitaxel resistance was partial in T47D-doxR, suggesting that HSF1 is more dominantly involved in drug resistance development of A549-taxolR than that of T47D-doxR. Moreover, the transfection of siHSF1 or S303/307A to A549-taxolR or T47D-doxR increased PAPR1 cleavage by paclitaxel treatment, and the levels were similar to those in A549 or T47D cells, respectively. In MTT assay, similar patterns were also shown (Fig. 6a, b).

\section{Correlation of pHSF1 at Ser303/307 with MDR1 expression} in spontaneously induced rat mammary tumor tissues and human lung cancer tissues

To elucidate the relationship between MDR1 and pHSF1 at Ser303/307 in mammary tumors, expressions of MDR1 and pHSF1 at Ser303/307 were examined using DMBAinduced rat mammary tumors. All rat mammary tumors 
A

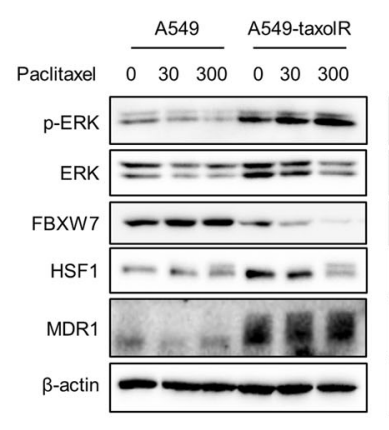

B

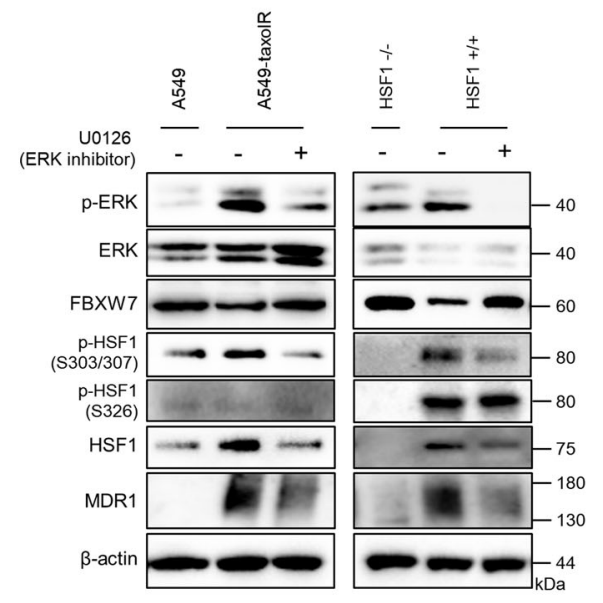

C

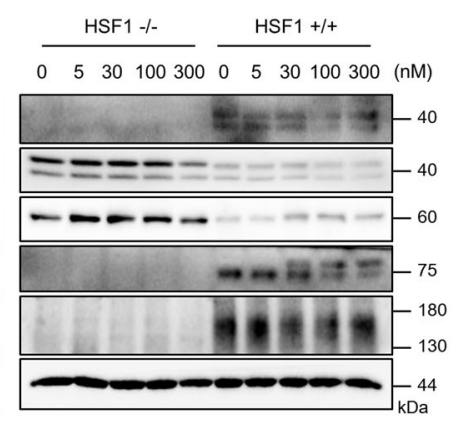

D
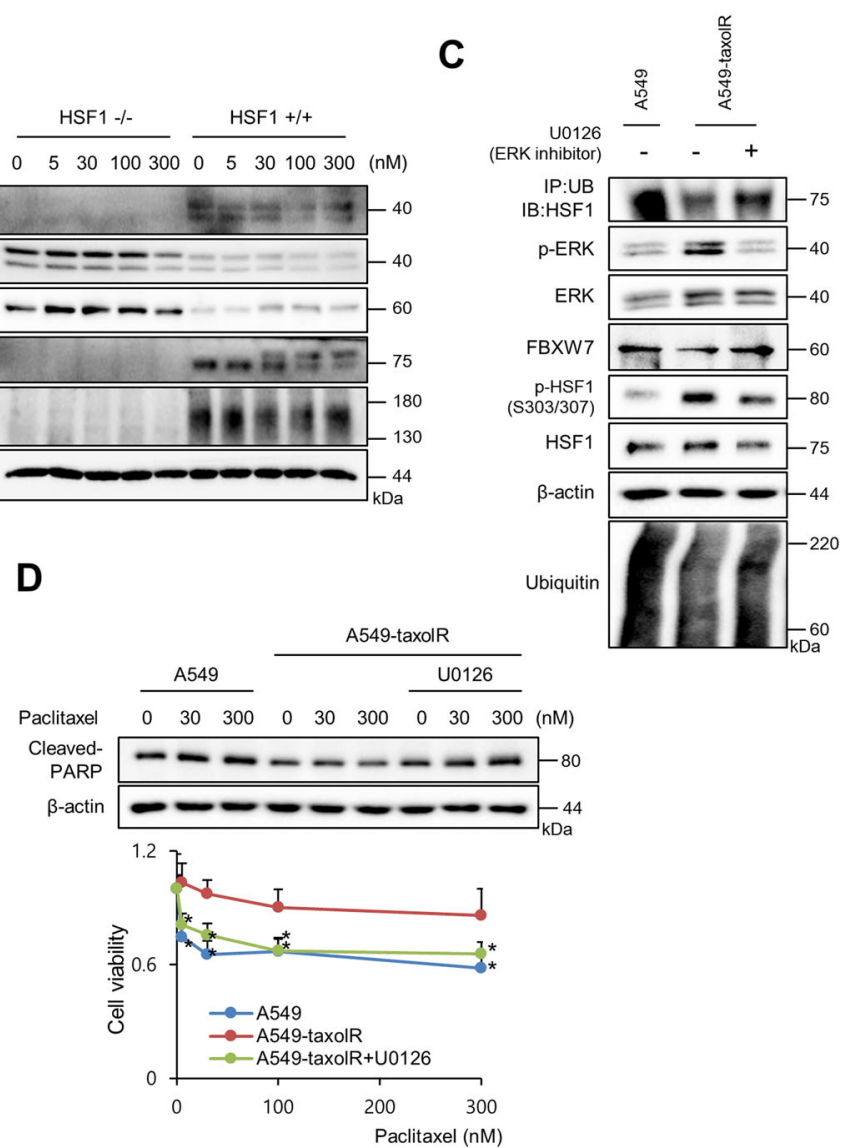

Fig. 5 ERK1/2 activation in drug-resistant cancer cells was involved in decreased FBXW7 expression. a Cells of A549, A549-taxolR, T47D, T47DdoxR, HSF1-/- mouse embryonic fibroblasts, and HSF1+/+ mouse embryonic fibroblasts were treated with indicated concentrations of paclitaxel for $24 \mathrm{~h}$, and western blotting was performed. b Cells of A549, A549-taxolR, HSF1-/- mouse embryonic fibroblasts, and HSF1+/+ mouse embryonic fibroblasts were treated with U0126, an ERK1/2 inhibitor (10 $\mu \mathrm{M})$, for $12 \mathrm{~h}$, and western blotting was performed. c Cell lysates of A549 and A549-taxolR with or without $\mathrm{U} 0126$ treatment $(10 \mu \mathrm{M})$ for $12 \mathrm{~h}$ were immunoprecipitated with a ubiquitin construct $(\mathrm{Ub})$ and immunoblotted with HSF1. Western blotting was also performed. $\mathbf{d}$ A549 and A549-taxolR cells were pretreated with or without U0126 treatment (10 $\mu \mathrm{M})$ for $1 \mathrm{~h}$ and were treated with indicated doses of paclitaxel. Cell death was analyzed by western blot analysis, and cell viability was determined by the MTT assay. Values are presented as percentages of cell survival in paclitaxel-treated cells relative to untreated cells and as the mean \pm SD of at least three independent experiments. Statistics calculated based on a Student's $t$-test, ${ }^{*} p<0.05$.

induced by DMBA were malignant adenocarcinomas. The immunofluorescence of pHSF1 at Ser303/307 (red) was co-localized in MDR1 expressed mammary tumors (green). Moreover, high expression of pHSF1 at Ser303/ 307 showed high levels of MDR1, and low expression of pHSF1 at Ser303/307 showed low level of MDR1, thus indicating a positive correlation (Fig. 7a). When human lung cancer tissue slides of 120 patient specimens were examined, similar expression patterns were observed (Fig. 7b), suggesting positive correlation between the expression of pHSF1 at Ser303/307 and MDR1.

\section{Discussion}

In this study, HSF1 was identified as an important factor in the transcriptional activation of $m d r 1$, which was finally identified to be involved in drug resistance. Drug-resistant cells of A549-taxolR, T47D-doxR, and MCF7-doxR showed overexpression of MDR1 at both mRNA and protein levels. Furthermore, the phosphorylation of HSF1 at Ser303/307, which are the characteristic posttranslational modification sites of HSF1, plays an important role in the transcriptional activation of $m d r 1$.

Paclitaxel- and doxorubicin-resistant cancer cells all showed resistance to paclitaxel and overexpression of $m d r 1$ at a transcriptional level, suggesting that the administration of different chemotherapeutic drugs increased $m d r 1$ gene and showed multidrug resistance. Interestingly, HSF1 was overexpressed, and paclitaxel treatment did not degrade HSF1 protein in drug-resistant cancer cells unlike in parent cells; this phenomenon was more dominant in paclitaxel-resistant cells, such as A549taxolR cells, than in doxorubicin-resistant cells, such as 


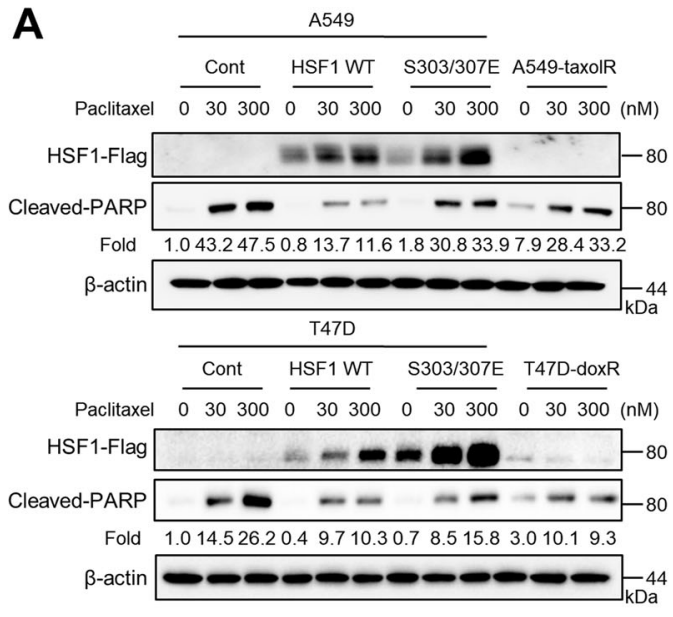

B

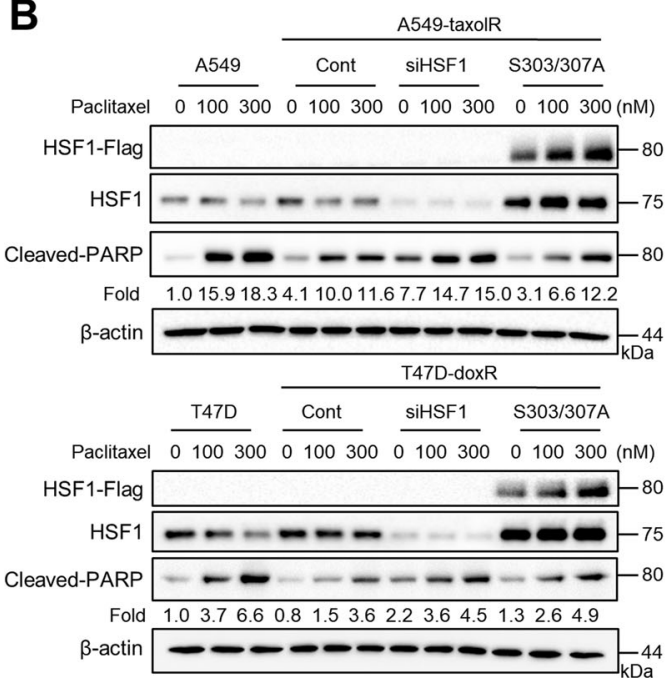

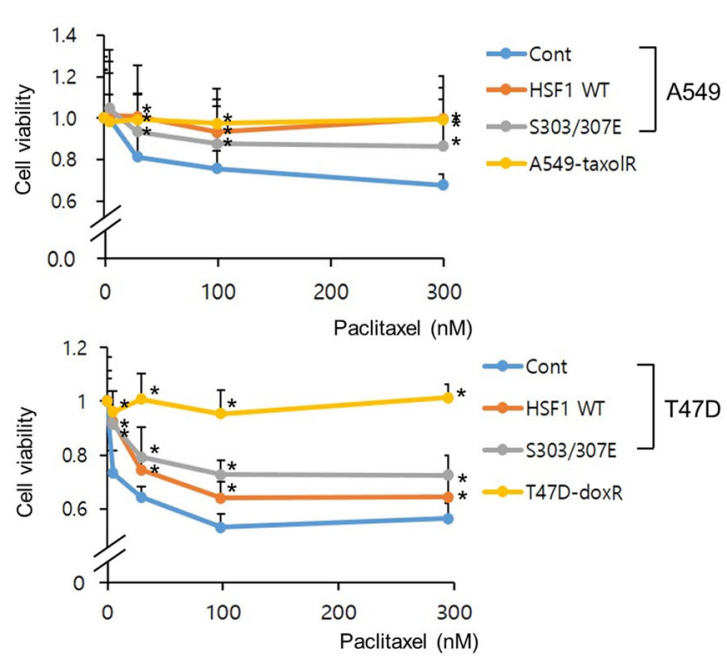
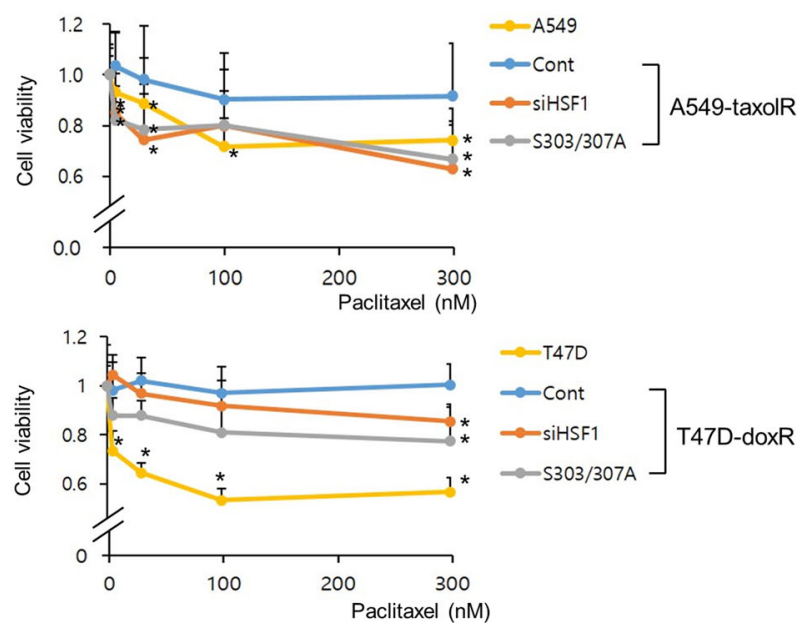

Fig. 6 Increased phosphorylation of HSF1 at Ser303/307 in drug-resistant cancer cells was responsible for paclitaxel resistance. A549, A549taxolR, T47D, and T47D-doxR cells after transient transfection of a WT-HSF1 and S303/307E, or b siRNA-HSF1 and S303/307A treated with indicated concentrations of paclitaxel for $24 \mathrm{~h}$. Western blotting was performed. Cell viability was determined by the MTT assay. Data are expressed as the mean \pm SD of at least three independent experiments. Protein levels were quantified using Image J software, and data are expressed as the fold change relative to the negative control. Statistics calculated based on a Student's $t$-test, ${ }^{*} p<0.05$ vs each control (Cont) of parent or resistant cells.

T47D-doxR cells. Since the mRNA level of $h s f 1$ was not altered, post-translational modification, such as phosphorylation, was examined, and it was found that pHSF1 at Ser303/307 was not degraded in drug-resistant cells. Regarding other phosphorylations of HSF1, such as those at Ser326 and Ser230, MDR1 expression was not related. The phosphorylation of HSF1 at Ser303/307 normally appears to repress the transactivation capacity of HSF1 for HSP transcription ${ }^{38}$ and is involved in cytoplasmic localization of HSF1 (ref. ${ }^{39}$ ). Indeed, the phospho-mimicking mutant S303/307E showed inhibited expression of HSP70 at both mRNA and protein levels; however, MDR1 expression was found to be increased. However, the phospho-defective mutant S303/307A showed the opposite trend, suggesting that pHSF1 at Ser303/307 acts as a partly inhibitory transcriptional factor of HSP expression under normal physiological growth conditions, whereas in the case of regulation of $m d r 1$ transcription during the drug resistance development process by continuous treatment with anticancer drugs, it acts as a transcriptional activator of $m d r 1$ expression. Since the $m d r 1$ gene has an HSE and it has been reported that cells overexpressing WT-HSF1 show increased expression of ABC transporters, including MDR1/ABCB1, and deletion of the HSF1 protein residues 221-315, which includes HSF1 phosphorylation sites S303/307, did not increase MDR1 expression $^{40}$, the phosphorylation of Ser303/307 may also have transactivating activity. Moreover, there are evidences of predominant nuclear localization of pHSF1 at Ser303/307 (ref. ${ }^{41}$ ), and our data also suggested 


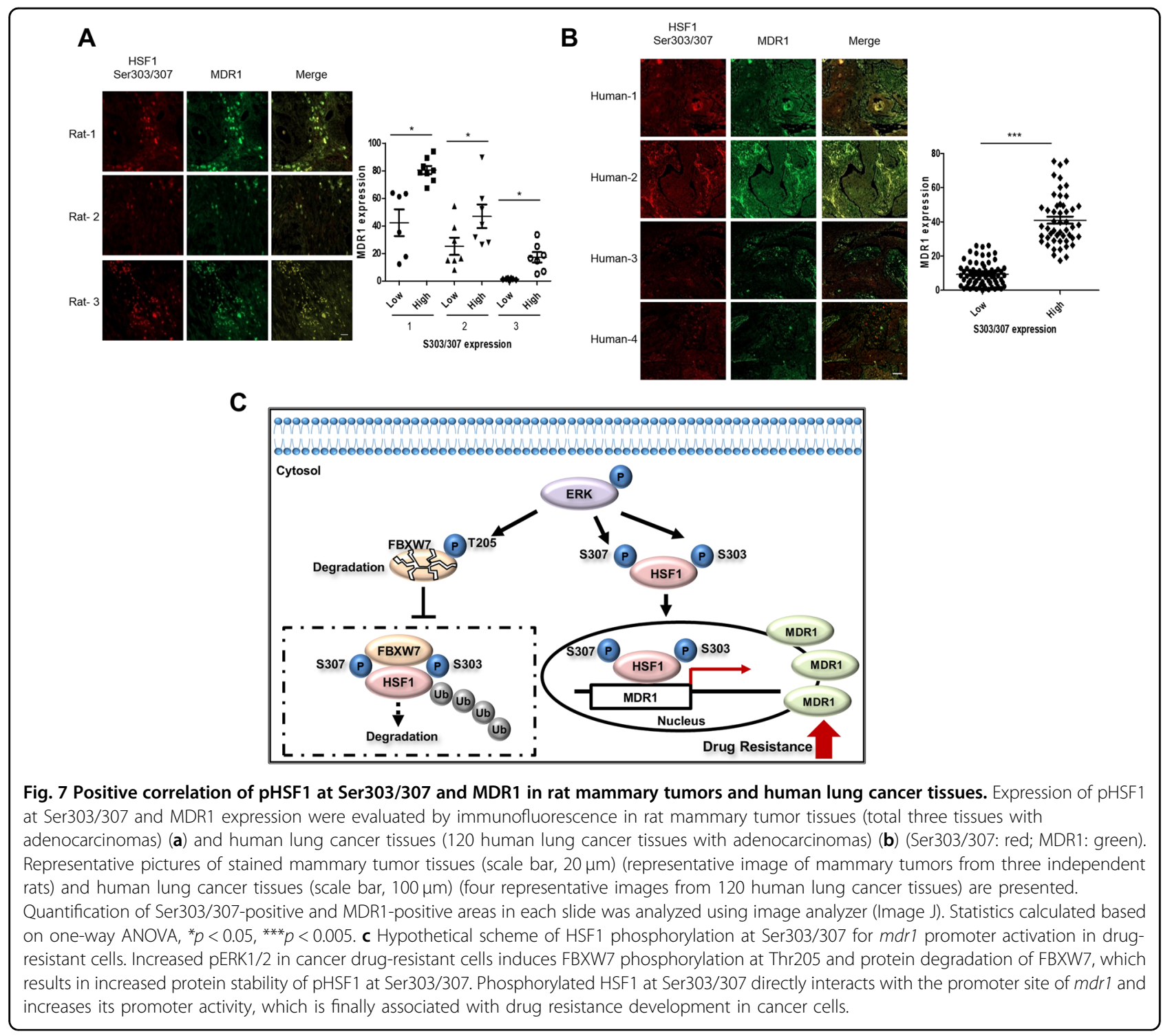

predominant nuclear localization of pHSF1 at Ser303/307. In this study, the activated form of HSF1 (pHSF1 at Ser326 and S230) for HSP induction did not exhibit the regulation of MDR1. Since the regulation of MDR1 is extremely complex and involves the interplay of various transcription factors depending on the circumstances of cells, the pHSF1 at Ser303/307 may interact with other transcription factors for $m d r 1$ transcription. However, this complexity and diversity in the regulation of MDR1 may add to the difficulty in finding the exact interplay with other factors.

Among the few papers that have been published on the relationship between HSF1 and MDR1, some suggested positive correlation between MDR1 and HSF1, wherein HSF1 up-regulated the expression of MDR $1^{4}$. However, other papers suggested that HSF1 down-regulated the expression of MDR1. The difference between the two systems was that positive regulation was examined using mice or human cancer cells and negative regulation was examined using non-cancer tissues of mice, such as cardiomyocytes and liver tissues. Mouse genomes contain two paralogous genes, $m d r l a$ and $m d r 1 b$, for P-glycoprotein, whereas humans have one $m d r 1$ gene ${ }^{42}$. The tissue distribution of $m d r 1 a / 1 b$ in mice is broad, including the liver and heart; however, the $m d r 1$ gene of humans is expressed at relatively low levels in normal conditions, and a stressed condition, such as treatment with chemotherapeutic drugs targeting cancer cells, can overexpress the $m d r 1$ gene $^{28}$. These different situations may differently regulate $m d r 1$ gene expression by HSF1.

The up-regulation of pHSF1 at Ser303/307 was reportedly degraded by the FBXW7 ubiquitin ligase, and our drug-resistant cells showed decreased FBXW7 expression. Because FBXW7 degraded pHSF1 at Ser303/ 
307 via ubiquitin degradation pathways, decreased expression of FBXW7 in drug-resistant cells may involve the inhibition of pHSF1 degradation. Indeed, knockdown of FBXW7 to parent cells or overexpression of FBXW7 to drug-resistant cells regulated pHSF1 at Ser303/307, while FBXW7 did not affect the phosphorylated form of Ser326, suggesting that the decreased expression of FBXW7 in drug-resistant cells specifically stabilized the pHSF1 at Ser303/307.

The phosphorylation of FBXW7 at T205 reportedly decreases FBXW7 protein stability ${ }^{43}$, and this phosphorylation was induced by ERK1/2 activation ${ }^{33}$. Drugresistant cells showed activation of ERK1/2 (refs. ${ }^{34-36}$ ) unlike their parent cells, which is key regulation of low expression of FBXW7 in drug-resistant cells. Indeed, treatment of ERK1/2 inhibitor to drug-resistant cells or phospho-defective mutants of FBXW7 at T205 (T205A) increased FBXW7 protein stability and degradation of pHSF1 at Ser303/307, which finally resulted in decreased expression of MDR1. These results suggested that ERK1/2 activation in drug-resistant cells phosphorylated FBXW7 at T205 and induced ubiquitin degradation of FBXW7, which inhibited the degradation of pHSF1 at Ser303/307 and promoted transcriptional activation of the $m d r 1$ gene.

Tissues from mammary tumors that were spontaneously induced by DMBA or from human lung cancers showed a co-localization of and positive correlation between pHSF1 at Ser303/307 and MDR1, suggesting the possibility that targeting pHSF1 at Ser303/307 will overcome MDR1-mediated drug resistance in cancer therapy (Fig. 7c).

Taken together, we show that decreased expression of FBXW7 in drug-resistant cells, which is mediated by ERK1/2 activation, specifically stabilized the pHSF1 at Ser303/307. Increased protein stability of pHSF1 promoted $m d r 1$ transcription by its direct interaction with the promoter site of $m d r l$ in drug-resistant cells, providing, for the first time, the post-translational modification of HSF1 for MDR1 regulation during drug resistance development.

\section{Materials and methods}

\section{Cell culture and treatments}

A549 human lung cancer cells, paclitaxel-resistant A549 cells (A549-taxolR), T47D human breast cancer cells, doxorubicin-resistant T47D cells (T47D-doxR), MCF7 human breast adenocarcinoma cells, and doxorubicinresistant MCF7 cells (MCF7-doxR) were maintained in RPMI-1640 medium and supplemented with $10 \%$ fetal bovine serum at $37^{\circ} \mathrm{C}$ in an incubator with a humidified atmosphere of $95 \%$ air and $5 \% \mathrm{CO}_{2}$. To maintain the paclitaxel resistance phenotype, A549-taxolR cells were maintained with occasional addition of $100 \mathrm{nM}$ paclitaxel (Santa Cruz Biotechnology). Paclitaxel-resistant A549- taxol cells were kindly provided by Prof. S.K. Lee (Seoul National University, Seoul, South Korea). Doxorubicinresistant T47D cells and doxorubicin-resistant MCF7 cells were kindly provided by Prof. Y.J. Kwon (Ewha Womans University, Seoul, South Korea). Wild type and hsf1 knockout mouse embryonic fibroblast (HSF1+/+ and HSF1 - / - MEF) cells were provided by Dr. Ivor J. Benjamin (University of Utah, Salt Lake City, UT). MEF (HSF1 $+/+$ and HSF1-/- MEF) cells were cultured in Dulbecco's minimal essential medium (DMEM) supplemented with heat-inactivated $10 \%$ fetal bovine serum (FBS) and antibiotics. Cell lines were tested by BioMycoX Mycoplasma PCR Detection Kit (JCBIO Co., Ltd) to ensure that they were mycoplasma-free. MG132 were purchased from Sigma Aldrich (St. Louis, MO, USA). A549 and A549-taxolR cells were treated with indicated concentrations of paclitaxel with or without MG132 $(10 \mu \mathrm{M})$ for $12 \mathrm{~h}$.

\section{Cell transfection}

Wild-type (WT) human HSF1 was cloned into p3XFLAG-Myc-CMV containing an N-terminal FLAGtag. The phosphorylation mutant HSF1 (S230A, S326E, S303/307A, and S303/307E) constructs were constructed using overlapped extension primers. Plasmids used in this study are listed in Supplementary Table S1. Pre-designed siRNA for human HSF1 (sc-35611) and a negative control siRNA $(30 \mathrm{nM})$ were purchased from Santa Cruz Biotechnology (Dallas, TX, USA). Pre-designed siRNA for $m d r 1$ was purchased from Bioneer (Daejeon, Korea). Transient transfection was carried out using Lipofectamine 2000 (Invitrogen), according to the manufacturer's guidelines. HSF1 shRNA (sc-35611-V), FBXW7 shRNA (sc-37547-V), polybrene (sc-134220), and sh-control plasmid (sc-10808) were obtained from Santa Cruz Biotechnology. To produce sh-control cells and sh-HSF1 cells, cell lines were selected using puromycin $(1 \mu \mathrm{g} / \mathrm{mL})$ for at least 1 week. Control CRISPR/Cas9 (Cont) plasmid (sc-418922) and HSF1 CRISPR/Cas9 knockout (KO) plasmid (sc-400432-KO-2) were purchased from Santa Cruz Biotechnology. For CRISPR/Cas9 KO system transfection, cells were seeded and transfected after $24 \mathrm{~h}$ using plasmid transfection medium (sc-108062) containing transfection reagent (sc-395739).

\section{MTT [3-(4,5-dimethylthiazol-2-yl)-2,5-diphenyltetrazolium bromide] assay}

Cell viability against paclitaxel-induced toxicity was determined using an MTT assay (Amersham Pharmacia Biotech) in 96-well plates. A549/A549-taxolR cells were seeded at a density of $2 \times 10^{4}$ cells/well in 96-well plates and treated with the desired concentration of paclitaxel $(0$, 5, 30, 100, and $300 \mathrm{nM}$ ) for $24 \mathrm{~h}$. The statistical significance was determined by the Student's $t$-test. The 
differences were considered significant if the $p$ value was less than 0.05 .

\section{$m d r 1$ promoter assay}

The MDR1-Luc plasmid was purchased from Addgene (Cambridge, MA, USA). mdr1 promoter activity was measured using a luciferase assay system kit (\#E4030), which was purchased from Promega (Madison, WI, USA). Protein was quantitated using a protein assay reagent (\#500-0006), which was purchased from Bio-Rad (Hercules, CA, USA).

\section{Cycloheximide chase assay}

FLAG stability was measured in the presence of cycloheximide (CHX, Sigma Aldrich) after transfection of S303/307A or S303/307E. At $24 \mathrm{~h}$ after transfection, the cells were split into multiple dishes, $\mathrm{CHX}$ was added $(10 \mu \mathrm{g} / \mathrm{mL})$, and the cells were harvested at the indicated times.

\section{Cell fractionation}

Cellular fractionation was performed using a subcellular protein fractionation kit (\#78840) purchased from Thermo Fisher Scientific (Waltham, MA, USA). The harvested cell pellets were resuspended in cytoplasmic extraction buffer and incubated at $4{ }^{\circ} \mathrm{C}$ for $10 \mathrm{~min}$ with gentle mixing. Samples were agitated every $5 \mathrm{~min}$ and then centrifuged at $500 \mathrm{~g}$ for $5 \mathrm{~min}$ to collect the cytoplasmic fraction. Pellets were resuspended, incubated in nuclear extraction buffer at $4{ }^{\circ} \mathrm{C}$ for $30 \mathrm{~min}$, and centrifuged at $5000 \mathrm{~g}$ for $5 \mathrm{~min}$ to obtain the nuclear fraction.

\section{ChIP assay}

A549 and A549-taxolR cells were fixed, nuclei were isolated, and chromatin was sheared by sonication using the ChIP Kit according to the manufacturer's instructions (ab500; Abcam). The sheared chromatin was immunoprecipitated using HSF1 antibody (ab-52757; Abcam) and IgG (ab500; Abcam). Details regarding the primers used in ChIP-qPCR can be found in Supplementary Table S2. The quantitative PCR conditions were as follows: $10 \mathrm{~min}$ at $95^{\circ} \mathrm{C}$, followed by 38 cycles of denaturation $(5 \mathrm{~s}$ at $\left.95^{\circ} \mathrm{C}\right)$, annealing $\left(10 \mathrm{~s}\right.$ at $\left.62^{\circ} \mathrm{C}\right)$, and extension $(20 \mathrm{~s}$ at $72^{\circ} \mathrm{C}$ ) with single acquisition of fluorescence at the end of each extension step. ChIP-qPCR was calculated as follows: fold enrichment $=2^{-((\mathrm{Ct} \text { IP })-(\mathrm{Ct} \text { mock }))}$. Mean values of three biological replicates were calculated. Statistical analysis was carried out using one-way ANOVA.

\section{Immunoblotting and immunoprecipitation}

For Immunoblotting, A549/A549-taxolR cells were seeded at a density of $3 \times 10^{5}$ cells/dish in $60-\mathrm{mm}$ cell culture dishes. After $24 \mathrm{~h}$ incubation, cells were treated with paclitaxel varying from 5 to $300 \mathrm{nM}$. Cells were harvested at certain time points $(6,12$, and $24 \mathrm{~h})$. For immunoprecipitation, cells $\left(1 \times 10^{6}\right)$ were lysed in immunoprecipitation buffer (50 mM HEPES, pH 7.6, $150 \mathrm{mM} \mathrm{NaCl}, 5 \mathrm{mM}$ EDTA, $0.1 \%$ Nonidet P-40). After centrifugation $(30 \mathrm{~min}$ at $15,000 \mathrm{~g}$ ) to remove particulate material, supernatants were incubated with antibodies (1:50) against ubiquitin with constant agitation at $4{ }^{\circ} \mathrm{C}$. Immunocomplexes were precipitated with protein $\mathrm{A} / \mathrm{G}$ PLUS-agarose (sc-2003; Santa Cruz Biotechnology) and analyzed by sodium dodecyl sulfate-polyacrylamide gel electrophoresis. Immunoblotting and immunoprecipitation were performed using the following antibodies: $\beta$-actin (sc-47778), HSP27 (sc-1048), pHSF1 (Ser230; sc30443-R), p-ERK (sc-7383), Lamin B1 (sc-374015), and ubiquitin (sc-8017) were purchased from Santa Cruz Biotechnology. HSF1 (ab-52757), pHSF1 (Ser326; ab76076), pHSF1 (Ser303/307; ab-81281), HSP70 (ab-1428), and FBXW7 (ab-109617) were purchased from Abcam (Cambridge, MA, USA). FLAG (\#F3165) was purchased from Sigma Aldrich. MDR1 (\#12683) and ERK (\#9101) were purchased from Cell Signaling (Danvers, MA, USA). Protein band intensity was visualized on ChemiDoc (BioRad) and quantified using Image J software 1.45 (National Institutes of Health, Bethesda, MD, USA).

\section{RT-PCR}

Total RNA was extracted using QUIazol (Quiazen), and cDNA was synthesized using the ReverTra Ace RT-PCR Kit (Toyobo). $m d r 1$ and gapdh transcript levels were measured by RT-PCR (GenDEPOT). GAPDH was used as an internal control gene. The detailed primer sequences for RT-PCR are provided in Supplementary Table S3.

\section{Immunofluorescent staining}

For cell immunofluorescence assays, cells were fixed with $10 \%$ paraformaldehyde, permeabilized with $0.1 \%$ Triton X-100 in phosphate-buffered saline (PBS), washed three times with PBS, and incubated with anti-HSF1 (Ser303/307) and anti-FLAG antibodies diluted 1:200 in PBS with $1 \%$ FBS overnight at $4{ }^{\circ} \mathrm{C}$. The cells were incubated with Alexa 568-labeled anti-rabbit (1:500) and Alexa 488-labeled anti-mouse (1:500) secondary antibodies. After they were washed three times with PBS, coverslips were mounted onto microscope slides using a mounting reagent (Southernbiotech, Birmingham, AL, USA). The slides were then analyzed using a ZEISS LSM 880 Confocal Laser Scanning Microscope (Carl Zeiss).

For breast tissue immunofluorescence assays, spontaneous mammary tumors were induced in female Sprague-Dawley (SD) rats by oral administration of DMBA (15 mg per rat; Sigma Aldrich). Rats were autopsied under anesthesia at 26 weeks after DMBA administration. Detailed experimental procedures have been published previously ${ }^{44}$. All animals were randomized but 
not performed blind experiments. Human lung cancer tissue slides were purchased from US Biomax. For antigen retrieval, slides were placed in citric acid buffer ( $\mathrm{pH}$ 6.0) and heated at $100{ }^{\circ} \mathrm{C}$ for $20 \mathrm{~min}$. Slides were coimmunostained with HSF1 (Ser303/307) (1:200) and MDR1 (1:200) overnight at $4{ }^{\circ} \mathrm{C}$. The slides were incubated with Alexa 568-labeled anti-rabbit (1:500) and Alexa 488-labeled anti-mouse (1:500) secondary antibodies. The nucleus was counterstained with DAPI (Sigma Aldrich), and the stained cells were imaged using a Zeiss Apotome (Carl Zeiss). Quantification of images was measured with image analyzer (Image J, NIH, Bethesda, MD, USA). All statistical analyses of images were performed using GraphPad Prism software 5.0 (GraphPad Software, San Diego, CA, USA).

\section{Statistical analysis}

Data expressed as mean \pm SD represented at least three independent experiments. Statistical significance was determined by Student's $t$-test or one-way ANOVA (Newman-Keuls test). The differences were considered significant if the $p$ value was $<0.05$. ANOVA test was performed using GraphPad Prism software 5.0.

\section{Acknowledgements}

This work was supported by grants from the National Research Foundation of Korea, (NRF-2020R1A2C3013255, NRF-2017M2A2A702019560, and NRF2018R1A5A2025286), funded by the Korean government (Ministry of Science and ICT).

\section{Data availability}

All data generated or analyzed during this study are included in this published article and its supplementary information files.

\section{Conflict of interest}

The authors declare that they have no conflict of interest.

\section{Publisher's note}

Springer Nature remains neutral with regard to jurisdictional claims in published maps and institutional affiliations.

Supplementary Information accompanies this paper at (https://doi.org/ 10.1038/s41419-020-2600-3).

Received: 13 November 2019 Revised: 7 May 2020 Accepted: 11 May 2020 Published online: 26 May 2020

\section{References}

1. Sreedhar, A. S. \& Csermely, P. Heat shock proteins in the regulation of apoptosis: new strategies in tumor therapy: a comprehensive review. Pharmacol. Ther. 101, 227-257 (2004).

2. Mosser, D. D. \& Morimoto, R. I. Molecular chaperones and the stress of oncogenesis. Oncogene 23, 2907-2918 (2004).

3. Ciocca, D. R. \& Calderwood, S. K. Heat shock proteins in cancer: diagnostic, prognostic, predictive, and treatment implications. Cell Stress Chaperones 10, 86-103 (2005).

4. Vilaboa, N. E., Galan, A., Troyano, A., de Blas, E. \& Aller, P. Regulation of multidrug resistance 1 (MDR1)/P-glycoprotein gene expression and activity by heat-shock transcription factor 1 (HSF1). J. Biol. Chem. 275, 24970-24976 (2000).
5. Kioka, N., Yamano, Y., Komano, T. \& Ueda, K. Heat-shock responsive elements in the induction of the multidrug resistance gene (MDR1). FEBS Lett. $\mathbf{3 0 1}$ 37-40 (1992).

6. Guettouche, T., Boellmann, F., Lane, W. S. \& Voellmy, R. Analysis of phosphorylation of human heat shock factor 1 in cells experiencing a stress. BMC Biochem. 6, 4 (2005).

7. Neef, D. W., Jaeger, A. M. \& Thiele, D. J. Heat shock transcription factor 1 as a therapeutic target in neurodegenerative diseases. Nat. Rev. Drug Discov. 10 930-944 (2011)

8. Santagata, S. et al. High levels of nuclear heat-shock factor 1 (HSF1) are associated with poor prognosis in breast cancer. Proc. Natl Acad. Sci. USA 108, 18378-18383 (2011)

9. Yeh, C. H., Bellon, M. \& Nicot, C. FBXW7: a critical tumor suppressor of human cancers. Mol. Cancer 17, 115 (2018).

10. Kourtis, N., Strikoudis, A. \& Aifantis, I. Emerging roles for the FBXW7 ubiquitin ligase in leukemia and beyond. Curr. Opin. Cell Biol. 37, 28-34 (2015).

11. Akhoondi, S. et al. FBXW7/hCDC4 is a general tumor suppressor in human cancer. Cancer Res. 67, 9006-9012 (2007).

12. O'Neil, J. et al. FBW7 mutations in leukemic cells mediate NOTCH pathway activation and resistance to gamma-secretase inhibitors. J. Exp. Med. 204, 1813-1824 (2007).

13. Thompson, B. J. et al. The SCFFBW7 ubiquitin ligase complex as a tumor suppressor in T cell leukemia. J. Exp. Med. 204, 1825-1835 (2007).

14. Kourtis, N. et al. FBXW7 modulates cellular stress response and metastatic potential through HSF1 post-translational modification. Nat. Cell Biol. 17, 322-332 (2015).

15. Gottesman, M. M. \& Pastan, I. Biochemistry of multidrug resistance mediated by the multidrug transporter. Annu. Rev. Biochem. 62, 385-427 (1993).

16. Chin, K. V., Tanaka, S., Darlington, G., Pastan, I. \& Gottesman, M. M. Heat shock and arsenite increase expression of the multidrug resistance (MDR1) gene in human renal carcinoma cells. J. Biol. Chem. 265, 221-226 (1990).

17. Kioka, N. et al. Quercetin, a bioflavonoid, inhibits the increase of human multidrug resistance gene (MDR1) expression caused by arsenite. FEBS Lett. 301, 307-309 (1992)

18. Kim, S. H. et al. Involvement of heat shock factor in regulating transcriptional activation of MDR1 gene in multidrug-resistant cells. Cancer Lett. 115, 9-14 (1997).

19. Kim, S. H. et al. Suppression of multidrug resistance via inhibition of heat shock factor by quercetin in MDR cells. Exp. Mol. Med. 30, 87-92 (1998).

20. Miyazaki, M. et al. Activation of human multidrug resistance-1 gene promoter in response to heat shock stress. Biochem. Biophys. Res. Commun. $\mathbf{1 8 7}$ 677-684 (1992).

21. Asakuno, $K$. et al. Involvement of a DNA binding protein, MDR-NF1/NB-1, in human MDR1 gene expression by actinomycin D. Biochem. Biophys. Res. Commun. 199, 1428-1435 (1994).

22. Ohga, $\mathrm{T}$. et al. Direct involvement of the Y-box binding protein YB-1 in genotoxic stress-induced activation of the human multidrug resistance 1 gene. J. Biol. Chem. 273, 5997-6000 (1998).

23. Gabai, V. L. \& Sherman, M. Y. Invited Review: Interplay between molecular chaperones and signaling pathways in survival of heat shock. J. Appl. Physiol. 92, 1743-1748 (2002).

24. Song, M., Pinsky, M. R. \& Kellum, J. A. Heat shock factor 1 inhibits nuclear factor- $\mathrm{KB}$ nuclear binding activity during endotoxin tolerance and heat shock J. Crit. Care 23, 406-415 (2008)

25. Reck, M. Heigener, D. F. Mok, T., Soria, J.-C. \& Rabe, K. F. Management of nonsmall-cell lung cancer: recent developments. Lancet 382, 709-719 (2013).

26. Holleman, A. et al. miR-135a contributes to paclitaxel resistance in tumor cells both in vitro and in vivo. Oncogene 30, 4386-4398 (2011).

27. Savaraj, N. et al. Multidrug-resistant gene expression in small-cell lung cancer Am. J. Clin. Oncol. 20, 398-403 (1997).

28. Kuo, M. T. Roles of multidrug resistance genes in breast cancer chemoresistance. Adv. Exp. Med. Biol. 608, 23-30 (2007).

29. Aldonza, M. B. et al. Suppression of MAPK signaling and reversal of mTORdependent MDR1-associated multidrug resistance by 21alphamethylmelianodiol in lung cancer cells. PLOS ONE 10, e0127841 (2015).

30. Jeon, K. H., Yu, H. V. \& Kwon, Y. Hyperactivated m-calpain affects acquisition of doxorubicin resistance in breast cancer cells. Biochim. Biophys. Acta Gen. Subj. 1862, 1126-1133 (2018).

31. Jun, K. Y., Park, S. E., Liang, J. L., Jahng, Y. \& Kwon, Y. Benzo[b]tryptanthrin inhibits MDR1, topoisomerase activity, and reverses adriamycin resistance in breast cancer cells. ChemMedChem 10, 827-835 (2015). 
32. Batista-Nascimento, L., Neef, D. W., Liu, P. C., Rodrigues-Pousada, C. \& Thiele, D. J. Deciphering human heat shock transcription factor 1 regulation via posttranslational modification in yeast. PLOS ONE 6, e15976 (2011).

33. Ji, S. et al. ERK kinase phosphorylates and destabilizes the tumor suppressor FBW7 in pancreatic cancer. Cell Res. 25, 561-573 (2015).

34. McCubrey, J. A. et al. Roles of the Raf/MEK/ERK pathway in cell growth malignant transformation and drug resistance. Biochim. Biophys. Acta 1773, 1263-1284 (2007).

35. Jeong, E. K. et al. Role of extracellular signal-regulated kinase (ERK)1/2 in multicellular resistance to docetaxel in MCF-7 cells. Int. J. Oncol. 37, 655-661 (2010).

36. Mirmohammadsadegh, A. et al. ERK1/2 is highly phosphorylated in melanoma metastases and protects melanoma cells from cisplatin-mediated apoptosis. J. Invest. Dermatol. 127, 2207-2215 (2007).

37. Wertz, I. E. et al. Sensitivity to antitubulin chemotherapeutics is regulated by MCL1 and FBW7. Nature 471, 110-114 (2011).

38. Chu, B., Zhong, R., Soncin, F., Stevenson, M. A. \& Calderwood, S. K. Transcriptional activity of heat shock factor 1 at 37 degrees $C$ is repressed through phosphorylation on two distinct serine residues by glycogen synthase kinase 3 and protein kinases Calpha and Czeta. J. Biol. Chem. 273, 18640-18646 (1998).
39. Wang, X., Grammatikakis, N., Siganou, A. \& Calderwood, S. K. Regulation of molecular chaperone gene transcription involves the serine phosphorylation, 14-3-3 epsilon binding, and cytoplasmic sequestration of heat shock factor 1 . Mol. Cell Biol. 23, 6013-6026 (2003).

40. Vydra, N., Toma, A., Glowala-Kosinska, M., Gogler-Piglowska, A. \& Widlak, W. Overexpression of heat shock transcription factor 1 enhances the resistance of melanoma cells to doxorubicin and paclitaxel. BMC Cancer 13, 504 (2013)

41. Hietakangas, V. et al. Phosphorylation of serine 303 is a prerequisite for the stress-inducible SUMO modification of heat shock factor 1. Mol. Cell Biol. 23, 2953-2968 (2003).

42. Devault, A. \& Gros, P. Two members of the mouse mdr gene family confer multidrug resistance with overlapping but distinct drug specificities. Mol. Cell Biol. 10, 1652-1663 (1990).

43. Min, S. $H$. et al. Negative regulation of the stability and tumor suppressor function of Fbw7 by the Pin1 prolyl isomerase. Mol. Cell. 46, 771-783 (2012).

44. Kim, S., Jin, H., Seo, H. R., Lee, H. J. \& Lee, Y. S. Regulating BRCA1 protein stability by cathepsin S-mediated ubiquitin degradation. Cell Death Differ. 26, 812-825 (2019). 